\title{
Block based Image Steganography using Entropy with LSB and 2-bit Identical Approach
}

\author{
Manoj Kumar \\ CE Dept. \\ Yadawindera College of Engineering \\ Talwandi Sabo,151302, Punjab, India
}

\author{
Gursewak Singh \\ Research Scholar, CE Dept. \\ Yadawindera College of Engineering \\ Talwandi sabo, 151302, Punjab, India
}

\begin{abstract}
Steganography plays a very imperative role in secret communication. Many possible techniques are used to embed confidential information in digital images, the least significant bit (LSB) technique has very widely used. In this paper the proposed technique a new steganography technique is being developed to hide data in image using pixel based algorithm. Also, to make the algorithm more undetectable data is divided into segments and image into blocks and a data segment is embedded into an image block where it effects. The least image quality. The experimental results prove that the quality of stego image using the proposed algorithm. For the verification of the results, peak signal-to noise (PSNR) and mean square error (MSE) are calculated.
\end{abstract}

\section{Keywords}

LSB; stego image; secret image; Arnold's transformation; identical bits

\section{INTRODUCTION}

Steganography is the process of concealing information in a carrier such as text, image, voice, video, or protocol. Digital images are one of the common and most popular ones due to their frequency on the Internet and high capacity of data transmission without degrading effect on images quality [1]. It is a high security technique for long data transmission. For many years it has been considered that security in cryptographic algorithms is directly related to the complexity of the mathematical operations that define the core of the encoding process. However, research using hardware-aided reverse engineering has continuously demonstrated that every cryptographic algorithm has a relatively short lifecycle, defined by the evolvement of computational power. A secure communication system is reliable as long as the cryptographic algorithm on top of which it was built is reliable [3].

In second section of this paper we have written related work to proposed technique and proposed work, experiment and results and conclusion are binded in sections 2, 3 and 4 respectively.

\section{2. LITERATURE REVIEW}

Harpal et.al [1] proposed a new steganography techniques for the colored image. Their are many steganography techniques like LSB,DCT, pixel based etc, but these technique have many problems. To improved technique used 2-2-4 LSB insertion three plans images and get best results.

R.kumar et.al [3] proposed a new method based on parity of the pixel in odd and even case. First of all message is encrypted by vernam cipher algorithm and apply LSB-S method of pixel and XOR operation, after this store the results and verify on different parameters to good results.
M.devi et al [3] proposed a new method based on parity of the pixel in odd and even case. First of all message is encrypted by vernam cipher algorithm and apply LSB-S method of pixel and XOR operation, after this store the results and verify on different parameters to good results.

\section{PROPOSED WORK}

In this section algorithm and techniques are explained which are used to apply for good results. In this paper we used a newly and best technique which is based on pixel value. Technique is explain below.

\section{2 bit-Identical}

This method is used to hide the secret message and pixel of the image bits are analyzed one by one if identical value finding between the pixel then hide the secret message on those bits.

\begin{tabular}{|c|c|c|c|c|c|c|c|}
\hline 1 & 0 & 0 & 1 & 1 & 0 & 0 & 1 \\
\hline & & & 1 & 1 & & & \\
\hline
\end{tabular}

Fig 1.2 bit Identical technique

\section{Least significant bit technique}

The most significant technique in steganography. It is used to hide the secret data in grey scale and colored images on its binary coding.fig 1.2 shows the LSB technique and shows the pixel of secret message bits. Algorithm shows the results by shifting right most two bits of LSBs of the pixel [1].

\begin{tabular}{|l|l|l|l|l|l|l|l|}
\hline 1 & 1 & 0 & 0 & 0 & 1 & 0 & 1 \\
\hline & & & & & & & \\
\hline
\end{tabular}

Fig 2. Least significant bit hiding technique

Entropy

There are many ways by which texture of digital image can be measured. Entropy is the most suitable way to measure the texture content of an image.Texture provides measure of properties of an image such as smoothness,coarseness and regularity.An image that is perfectly flat will have entropy of zero.The area of the image which have higher values of entropy are least visible to human because human eye is insensitive to these high entropy areas.Hence, if secrect image 
is embedded in high entropy areas of an image then higher imperceptibility can be obtained.Entropy can be defined as the statistical measure of randomness.

$$
\mathrm{E}=-\sum P \log _{2}(P)
$$

Where $\mathrm{E}$ is the entropy and $\mathrm{P}$ is the histogram count.

\section{ARNOLD'S CAT MAP}

Arnold's cat map (ACM) or Arnold transform (AT), proposed by Vladimir Arnold in 1960, is a chaotic map which when applied to a digital image randomizes the original organization of its pixels and the image becomes Imperceptible or noisy. However, it has a period $\mathrm{p}$ and if iterated $\mathrm{p}$ number of times, the original image reappears [11].

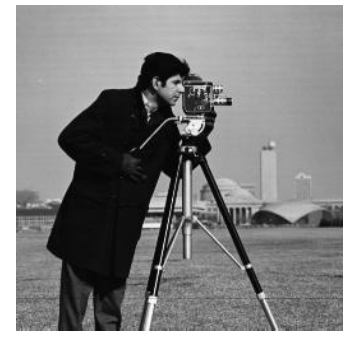

(a)

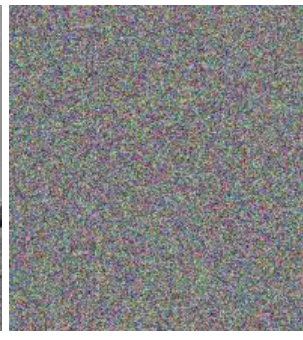

(b)

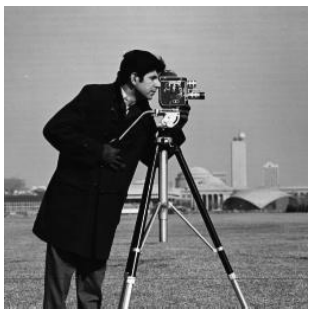

(C)

Fig 3. (a) original secret image.(b)encrypted secret image.(c)decrypted image

In this figure shows that having original image encrypted with secret image apply ACM random iteration of 20 times which obtain an encrypted image. we can retrieve the original image by applying inverse Arnold'stransformation.

Table. 1 Steps of Hiding Process

\begin{tabular}{|l|l|}
\hline 1 & Read 256x256 color cover image. \\
\hline 2 & $\begin{array}{l}\text { Divide color image into 16 images } \\
\text { into 64x64 bits. }\end{array}$ \\
\hline 3 & $\begin{array}{l}\text { Select four blocks of image to hide } \\
\text { data. }\end{array}$ \\
\hline 4 & Find RGB of each of four blocks. \\
\hline 5 & $\begin{array}{l}\text { Convert green component of each } \\
\text { block into bits, } 64 \times 64 \times 8=32768 .\end{array}$ \\
\hline 6 & $\begin{array}{l}\text { Divide these bits into } 4096 \text { groups of } \\
8 \text { bits each. }\end{array}$ \\
\hline
\end{tabular}

\begin{tabular}{|l|l|}
\hline 7 & $\begin{array}{l}\text { Read 64x64 grey scale secret image } \\
\text { and apply arnold's transformation to } \\
\text { get an encrypted image }\end{array}$ \\
\hline 8 & $\begin{array}{l}\text { Convert encrypted image into bits } \\
64 \times 64 \times 8=32768 .\end{array}$ \\
\hline 9 & $\begin{array}{l}\text { Divide these bits into 4 blocks having } \\
8192 \text { bits each then in these groups } \\
\text { divide bits into 4096 groups of 2 bit } \\
\text { each. }\end{array}$ \\
\hline 10 & $\begin{array}{l}\text { Select group of bits of block 1 we } \\
\text { have choosen for hiding data and in } \\
\text { first group of bits of secret image, } \\
\text { apply proposed algorithm.(2-bit } \\
\text { identical+LSB),save bit positions in } \\
\text { an array(4 arrays for 4 blocks). }\end{array}$ \\
\hline 11 & $\begin{array}{l}\text { Repeat step 10 all of four blocks we } \\
\text { have selected for hiding data. }\end{array}$ \\
\hline 12 & $\begin{array}{l}\text { Combine all of 16 blocks of image } \\
\text { (12 blocks + 4 updated blocks). }\end{array}$ \\
\hline 13 & $\begin{array}{l}\text { Combine all of 16 blocks of image(12 } \\
\text { blocks + 4 updated blocks). }\end{array}$ \\
\hline
\end{tabular}

Table. 2 Steps of Retreving process

\begin{tabular}{|c|c|}
\hline 1 & $\begin{array}{l}\text { First of all Read the stego image of } \\
256 \times 256 \text { bits. }\end{array}$ \\
\hline 2 & $\begin{array}{l}\text { Divide the stego image into } 16 \text { blocks of } \\
\text { each bits. }\end{array}$ \\
\hline 3 & $\begin{array}{l}\text { Then select } 4 \text { blocks from where we } \\
\text { have already fixed. }\end{array}$ \\
\hline 4 & Apply RGB on each of 4 blocks of bits. \\
\hline 5 & $\begin{array}{l}\text { Convert RGB's green componet of } \\
64 \times 64[1 \times 8=32768\end{array}$ \\
\hline 6 & $\begin{array}{l}\text { Divide each of the blocks with } 8 \text { bits } \\
\text { which is }=4096 \text {. }\end{array}$ \\
\hline 7 & Repeat 5,6 step until for each 4 blocks. \\
\hline 8 & $\begin{array}{l}\text { Select first group and having an array } \\
\text { that was defined in first embedding } \\
\text { process. }\end{array}$ \\
\hline 9 & $\begin{array}{l}\text { Select position of bits from an array and } \\
\text { extract at those positions from } \\
\text { corresponding } \\
\text { Blocks }\end{array}$ \\
\hline 10 & Repeat the 8,9 steps each of 4 block. \\
\hline 11 & $\begin{array}{l}\text { After this combine those extracted bits } \\
\text { to get encrypted secret image. }\end{array}$ \\
\hline 12 & $\begin{array}{l}\text { Apply ARNOLD'S inverse } \\
\text { transformation, shows the extracted } \\
\text { image. }\end{array}$ \\
\hline
\end{tabular}




\section{EXPERIMENTAL RESULTS}

The proposed technique has been implemented for cover image. With the help of this stego image has been evaluetad. For implementation we have several colored cover images of $256 \times 256$ pixel then divide into different blocks of each pixel of $64 \times 64$ total 16 blocks. Grey scale image of $64 \times 64 \times 8$ bits. Then proposed scheme presented digital image that employ hybrid technique of 2-bit identical. PSNR Peak Signal Noise Ratio is used to measure the quality between cover image and stego image within size.

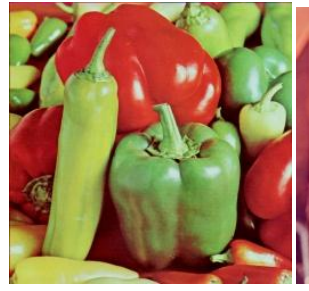

(a)

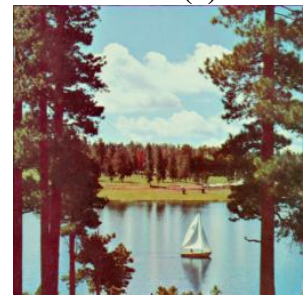

(c)

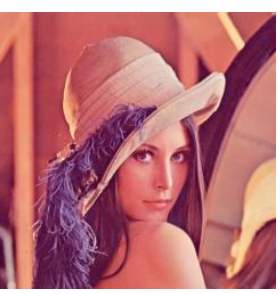

(b)

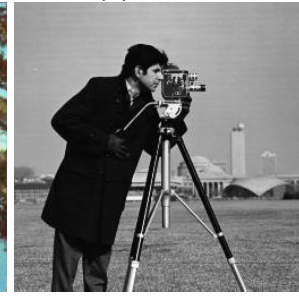

(d)
Fig 4. (a)Peeper (b) lena (c) lake (d) cameraman.

\section{Peak Signal Noise Ratio}

PSNR is used to measure the quality between cover image and stego image within size.

PSNR $=10 \cdot \log _{10}\left(\frac{\mathrm{MAX}_{\mathrm{i}}^{2}}{\mathrm{MSE}}\right)$

In this formula I is the value of each pixel. Greater the value of PSNR better the quality of image.

MSE ( Mean Square Error) also a parameter used to test the performance of proposed algorithm.

MSE $=\frac{1}{\operatorname{Min}} \sum_{0}^{\mathrm{m}-1} \sum_{0}^{\mathrm{n}-1}\|\mathrm{f}(\mathrm{i}, \mathrm{j})-\mathrm{g}(\mathrm{i}, \mathrm{j})\|^{2}$

where, $\mathrm{M}$ and $\mathrm{N}$ denotes the total number of pixels in the horizontal and vertical dimensions of an image. Xij represents the pixels in original image and Yij represent the pixels of stego image.

\section{Imperceptibility}

To measure the imperceptibility of proposed method. PSNR and MSE are calculated for different data bits.
Table 3 Results are shown in the table.

\begin{tabular}{|c|c|c|c|c|}
\hline Images & Original image & Stego image & PSNR & MSE \\
\hline Peeper & & & $\begin{array}{l}62.266 \\
1\end{array}$ & $\begin{array}{l}0.011 \\
4\end{array}$ \\
\hline Babbon & & 1 & $\begin{array}{l}61.671 \\
1\end{array}$ & $\begin{array}{l}0.011 \\
63\end{array}$ \\
\hline lena & & & $\begin{array}{l}62.670 \\
8\end{array}$ & $\begin{array}{l}0.003 \\
1\end{array}$ \\
\hline lake & & & $\begin{array}{l}61.660 \\
7\end{array}$ & $\begin{array}{l}0.021 \\
1\end{array}$ \\
\hline home & & & 61.936 & $\begin{array}{l}0.014 \\
7\end{array}$ \\
\hline
\end{tabular}

The hiding capacity of proposed method is high up to $2 \mathrm{bpp}$. Several images of $256 \times 256$ pixels are taken and data is hidden in these pixels. The total capacity of the proposed method is 131072 bits.

The capacity is calculated as given below:

capacity of 1 blocks $=64 \times 64 \times 2=8192$

capacity of 4 blocks $=64 \times 64 \times 2 \times 4=32768$

capacity of 16 blocks $=64 \times 64 \times 2 \times 16=131,072$

Total capacity $=131,072$.

\section{CONCLUSION}

In above proposed scheme using 2-bit identical approach with LSB, we have achieved PSNR value above 60 for different images shown in table which implies that our proposed scheme results in good perceptual quality of stego image. Arnold's transformation provides security by encrypting the secret image. For future work, it can be extended to be used with other techniques like DHT, DWT etc. Further it can be extended for video steganography.

\section{FUTURESCOPE}

At some point LSB seemed to be unbreakable but as natural images were better understood and newer models were created LSB gave way to new and more powerful algorithms which try to minimize changes to image statistics. But with further improvement in understanding of the statistical regularities and redundancies of natural images, most of these algorithms have also been successfully steganalysed. For future work, it can be extended to be used with other techniques like DHT, DWT etc. Further it can be extended for video Steganography. 


\section{ACKNOWLEDGMENTS}

First of all, I am extremely grateful to my Research Guide, Er. Manoj Kumar, Assistant Professor, for his valuable guidance, scholarly inputs and consistent encouragement. A person with an amicable and positive disposition, Sir has always made his available to clarify my doubts despite his busy schedules and I consider it as a great opportunity to work on my thesis under his guidance.

\section{REFERENCES}

[1] A.singh, H.singh, "An Improved LSB based Image Steganography Technique for RGB Images," international confrence IEEE, vol.978-1-4799-60859/15/\$31.00 @2015 IEEE.

[2] M.devi, N.sharma, "Improved detection of LSB SteganographyAlgorithms in Color and Gray Scale Images," UIET Panjab University Chandigarh, vol. 978$1-4799-2291-8 / 14 / \$ 31.00$ @2014 IEEE.
[3] K.joshi, R.kumar, "A New LSB-S Image Steganography Method Blend with Cryptography for Secret Communication", Third International Conference. Vol. 978-1-5090-0148-4/15/\$31.00@ 2015 IEEE .

[4] Shashikala Channalli, Ajay Jadhav , "Steganography An Art of Hiding Data", International Journal of Computer Science and Engineering.

[5] C.-K. Chan and L. Cheng, "Hiding data in images by simple $\{\mathrm{LSB}\} \mathrm{g}$ substitution ," Pattern Recognition, vol. 37, no. 3, pp. $469-474,2004$.

[6] X. Qing., X. Jianquan and X. Yunhua., "A High Capacity Information Hiding Algorithm in Color Image.", Proceedings of 2nd International Conference on EBusiness and Information System Security, IEEE Conference Publications, pp 1-4, 2010. 